Published in final edited form as:

Nat Neurosci. 2010 March ; 13(3): 369-378. doi:10.1038/nn.2501.

\title{
Stimulus onset quenches neural variability: a widespread
}

Mark M Churchland ${ }^{1,2,16}$, Byron $\mathbf{M} \mathbf{Y u}^{1,2,3,16}$, John P Cunningham ${ }^{1}$, Leo P Sugrue ${ }^{2,4}$, Marlene R Cohen ${ }^{2,4}$, Greg S Corrado ${ }^{2,4}$, William T Newsome ${ }^{2,4,5}$, Andrew M Clark ${ }^{6}$, Paymon Hosseini ${ }^{6}$, Benjamin B Scott ${ }^{6}$, David C Bradley ${ }^{6}$, Matthew A Smith ${ }^{7}$, Adam Kohn ${ }^{8,9}$, J Anthony Movshon ${ }^{9}$, Katherine M Armstrong ${ }^{2,5}$, Tirin Moore ${ }^{2,5}$, Steve W Chang ${ }^{10}$, Lawrence Stephen I Ryu 14, Gopal Sant

${ }^{3}$ Howard Hughes Medical Institute, Stanford University, Stanford CA, 94305, USA.

Department of Neurobiology, Stanford University School of Medicine, Stanford University

Department of Neurosurgery, Stanford University School of Medicine, Stanford University, Department of Psychology and Committee on Computational Neuroscience, University of Pittsburgh, Pittsburgh, PA, USA Department of Neuroscience, Albert Einstein College of Medicine, Bronx, NY, USA.

Users may view, print, copy, download and text and data- mine the content in such documents, for the purposes of academic research, subject always to the full Conditions of use: http://www.nature.com/authors/editorial_policies/license.html\#terms

Correspondence should be addressed to M.M.C. (church@stanford.edu).

${ }^{16}$ These authors contributed equally to this work.

Author Contributions. MMC and BMY contributed equally to this work. MMC wrote the manuscript, performed the FF and FA analyses, and made the figures. GPFA was developed by BMY, JPC, MS and KVS. This application of FA was devised by MMC and BMY. The mean-matched Fano factor was developed by MMC and KVS. The conception for the study arose from conversations between MMC, KVS, BMY, DCB, MRC, WTN and JAM. V1 data (extracellular) were collected in the laboratory of JAM by MAS and AK and in the laboratory of AK. V4 data were collected in the laboratory of TM by KMA. MT (plaid) data were collected in the laboratory of DCB by AMC, PH and BBS. MT (dots) data were collected in the laboratory of WTN by MRC. LIP and OFC data were collected in the laboratory of WTN by LPS, using an experimental design developed by LPS and GSC. PRR data were collected in the laboratory of LHS by SWC. PMd data were collected in the laboratory of KVS by BMY, SIR, GS and MMC. MT (direction/area and speed) data were collected in the laboratory of Stephen G. Lisberger by NJP and MMC. Intracellularly-recorded V1 data were collected by NJP and IMF in the laboratory of DF. All authors contributed to manuscript revisions and editing, particularly JAM, WTN, LPS, DF, JPC, BMY and KVS. 
${ }^{11}$ Howard Hughes Medical Institute, W.M. Keck Foundation Center for Integrative Neuroscience and Department of Physiology, University of California San Francisco, San Francisco, California, USA.

${ }^{12}$ Section of Neurobiology, School of Biological Sciences, University of Texas at Austin, Austin, Texas, USA.

${ }^{13}$ Department of Neurobiology and Physiology, Northwestern University, Evanston, Illinois, USA.

${ }^{14}$ Department of Neurosurgery, Stanford University School of Medicine, Stanford University, Stanford, California, USA.

${ }^{15}$ Department of Bioengineering, Stanford University, Stanford, California, USA.

\section{Abstract}

Neural responses are typically characterized by computing the mean firing rate. Yet response variability can exist across trials. Many studies have examined the impact of a stimulus on the mean response, yet few have examined the impact on response variability. We measured neural variability in 13 extracellularly-recorded datasets and one intracellularly-recorded dataset from 7 areas spanning the four cortical lobes. In every case, stimulus onset caused a decline in neural variability. This occurred even when the stimulus produced little change in mean firing rate. The variability decline was observable in membrane potential recordings, in the spiking of individual neurons, and in correlated spiking variability measured with implanted 96-electrode arrays. The variability decline was observed for all stimuli tested, regardless of whether the animal was awake, behaving, or anaesthetized. This widespread variability decline suggests a rather general property of cortex: that its state is stabilized by an input.

A fundamental approach of systems neuroscience is to probe the brain with repeated stimulus 'trials' and infer neural mechanism from the recorded responses. Extracellularlyrecorded responses are typically analyzed by computing the average spike rate across trials. By averaging, the experimenter hopes to overcome the apparent noisiness of spiking and estimate the true change in the neuron's underlying firing rate. It is likely true that much of the recorded spiking variability is effectively noise, and doesn't reflect fundamentally different responses on different trials. Yet it is nevertheless clear that the neural response can vary meaningfully across trials. For example, the neural state may be initially similar across trials, but become variable in response to a stimulus, as in1. Alternately, sensory cortex can be restless and active 2 prior to stimulus onset. A central question is whether the stimulusdriven response suppresses such ongoing variability3,4,5, superimposes with it2,6,7, or yields even greater variability due to non-linear interactions8? In general, does stimulus onset drive variability up (due to the variable responses themselves) or down (due to suppression of variable ongoing activity)?

In general, the mean rate provides an incomplete characterization of the neural response. A fuller characterization requires - at the least - knowing whether rate variability is present and how it changes with time. For example, the responses in Figure 1a and $\mathrm{b}$ have similar means, yet one would infer different things about the neural circuits that gave rise to them. The mean in Figure 1c erroneously suggests little stimulus-driven response. Supplementary 
Figure 1 illustrates a similar scenario using a simulated network. Because such situations may be common, it is important to characterize not only the stimulus-driven change in mean rate, but also the stimulus-driven change in rate variance.

The impact of a stimulus on variability could, of course, depend on the brain area, stimulus, and task. However, stimulus onset reduces both membrane potential variability in anaesthetized cat V13,4 and firing-rate variability in premotor cortex of reaching monkeys 9,10 . The presence of similar effects in two very different contexts suggests that a decline in variability could be a common feature of the cortical response. This would agree with recent theoretical work11,12 indicating that such an effect may be a general property of large recurrent networks.

To address this issue, we analyzed recordings from many cortical areas, driven via a variety of stimuli. A measure of firing-rate variability (the Fano factor) revealed a stimulus-driven decline in variability that was similar in timecourse to the decline in V1 membrane-potential variability. This decline was present not only for anaesthetized V1, but for all cortical areas tested regardless of the stimulus or behavioral state. The decline was also present in the correlated firing-rate variability of neurons recorded using implanted multi-electrode arrays. Finally, we demonstrate how recently developed methods, applied to simultaneous multielectrode recordings, can reconstruct the variable evolution of firing rates on individual trials.

\section{Results}

\section{Across-trial variability in the membrane potential}

Stimuli and task events can alter the structure and correlation 13 of membrane-potential variability. In particular, visual stimuli drive a reduction in membrane potential $\left(\mathrm{V}_{\mathrm{m}}\right)$ variability in cat primary visual cortex (V1) that is largely independent of stimulus orientation3,4. We re-analyzed data from4 to illustrate the timecourse of this effect (Fig. 2). Stimulus onset drives an immediate decline in $\mathrm{V}_{\mathrm{m}}$ variability. This decline occurs even for non-preferred stimuli that elicit little change in mean $V_{m}$ (see also3 and Fig. 7c,d of 4). Average variability (across all neurons/conditions) declined rapidly following stimulus onset and then remained at a rough plateau (Fig. 2c). The variability in question is across-trial variability, with a fairly long autocorrelation. When $\mathrm{V}_{\mathrm{m}}$ is low (or high), it tends to stay low (or high) for 10's to 100's of ms.

The relationship between intracellularly-recorded $V_{m}$ variability and extracellularlyrecorded firing-rate variability is likely to be complex, given the nonlinear and dynamic relationship between $\mathrm{V}_{\mathrm{m}}$ and firing rate (e.g., considerable $\mathrm{V}_{\mathrm{m}}$ variability occurs below threshold). One nevertheless expects across-trial $V_{m}$ variability to produce across-trial firing rate variability. Consistent with this expectation, firing-rate variability declines in parallel with $\mathrm{V}_{\mathrm{m}}$ variability, as we will show below. A larger question is whether the observed decline in variability is specific to $\mathrm{V} 1$ or whether it reflects a broader phenomenon. The latter is suggested both by the presence of a similar effect in a premotor cortex 9 and by recent theoretical work11,12. 
Addressing this issue requires quantifying firing-rate variability in extracellular recordings. While quantifying $\mathrm{V}_{\mathrm{m}}$ variability is straightforward, quantifying firing-rate variability is more complicated. Extracellularly-recorded spike trains are usually described in terms of an underlying firing rate (often termed $\lambda$ ) observed via a noisy point process (e.g., Poisson) that produces spikes. It should be stressed that this conception captures the statistics of neurons embedded in a network14,15: spike generation at the axon hillock in not responsible for the noisy spiking-process statistics 16 , nor is firing rate synonymous with membrane-potential. Rather, the underlying firing rate can be thought of as the average response of many similarly tuned neurons, or as the average response of one neuron across truly identical trials. Of course, repeated trials are not guaranteed to be truly identical: the underlying firing rate may differ somewhat. It is precisely this variability that we wish to capture, while ignoring variability arising from the roughly-Poisson spiking. Spiking variability may have interesting structure of its own, but for present purposes acts as noise.

Poisson spiking-process 'noise' can severely mask underlying firing-rate variability (Supplementary Fig. 1). It is thus rarely possible to discern changes in firing-rate variability by eye. We use two approaches to isolate underlying firing-rate variability from the variability contributed by spiking noise. The first approach employs a modified method for computing the Fano factor. This method is applicable to conventionally recorded singleneuron data, allowing analysis of a large number of existing datasets. The second approach uses factor analysis to assess covariance in large-scale simultaneous recordings. These methods are technically very different, but both are intended to assess the same thing: the degree of across-trial firing rate variability, independent of the contribution of noisy spiking statistics.

\section{A variability decline across multiple cortical areas}

We first employed the Fano factor (FF): the spike-count variance divided by the spike-count mean. Counts were made in a sliding window over the duration of the trial. The FF has been used extensively to characterize neural variability (e.g.,17,18,19). The FF is influenced both by variability arising from spiking noise, and by across-trial variability in the underlying firing rate20. Most prior work assumes that the underlying firing rate is similar across trials, and uses the FF to assess the statistics of spiking noise, which are roughly Poisson ( $\mathrm{FF} \approx 1$ ) for most of cortex. In the present work, we begin with the assumption that spiking noise is roughly Poisson, and we use the FF to assess across-trial variability in the underlying rate. An FF greater than one will be interpreted as an indication of across-trial firing-rate variability. Changes in the FF will be interpreted as reflecting changes in across-trial firingrate variability $9,20,21$. Although this approach begins by assuming Poisson spiking noise, it is reasonably robust to violations of that assumption (it is sufficient that spiking-noise variance scale linearly with the mean; the slope needn't be unity). We will also present critical controls for potential artifacts related to non-Poisson spiking noise. But before doing so we examine how the FF behaves across a variety of cortical areas.

We computed the mean firing rate and the FF for ten datasets from seven cortical areas of the macaque monkey (Fig. 3): V1, V4, MT, the lateral intra-parietal area (LIP), the parietal reach region (PRR), dorsal premotor cortex (PMd) and orbitofrontal cortex (OFC). 
Responses were to various visual stimuli or, for OFC, to juice reward. For each area the FF was averaged across neurons/conditions (see below). This is similar to what was done for the membrane potential analysis, and reflects both a desire for statistical power and the expectation that variability may change for both preferred and non-preferred stimuli (as in Fig. 2a,b).

In every case, stimulus onset drove a decline in firing-rate variability as assessed by the FF (all p-values <0.02). This is remarkable, given the diversity of areas, stimuli, and behavioral states. Variability declined during responses to simple visual stimuli, during operantlyconditioned responses (PRR and PMd), and during reward-driven responses (OFC). The variability decline was present regardless of whether the animal was anaesthetized (V1 and the MT datasets at bottom), passively viewing (V4) or performing a task (the other six datasets). For the MT datasets at bottom, stimulus onset occurred in two stages: pattern onset followed by motion onset. Both events drove a decline in variability, although only the more effective moving stimulus drove a sustained decline.

We have previously proposed that declining variability in premotor cortex is related to the progress motor preparation9. The changes in V1 membrane potential variability have been linked to shunting inhibition 3 and contrast-invariant orientation tuning4. These explanations are no less likely given the current data. Yet it is clear that the decline in variability is not limited to those cases, but constitutes a cortex-wide effect. This suggests a very general property of cortex: that its state is stabilized by an input.

\section{The mean-matched Fano factor controls for rising rates}

The FF declines as mean firing rates are rising. This is expected: in the intracellular data it was similarly true that $\mathrm{V}_{\mathrm{m}}$ variability dropped as the mean $\mathrm{V}_{\mathrm{m}}$ rose. It is nevertheless critical to rule out any possibility that the declining FF is trivially related to rising rates. At higher rates, refractory periods will tend to regularize spiking (a violation of the Poisson assumption) which could reduce the FF simply because the variability of spiking-process noise is reduced. A less obvious concern is that across-trial firing-rate variability could be constant, but becomes normalized by a higher mean after stimulus onset (the FF is the spikecount variance divided by the mean).

To address these issues, the FF traces above (Fig. 3) were computed using a method we term 'mean-matching.' The motivation is as follows: interpretation would be aided if we could record from a population of neurons that did not change its overall distribution of mean firing rates. If some neurons responded to the stimulus with an increased mean rate, and others with a decreased mean rate, then the overall distribution of mean rates (across neurons and conditions) could stay conveniently constant. For many areas (e.g., PRR) this is not far from what was observed. The overall mean rate always increased post-stimulus, yet this was often driven by a small percentage of neurons that strongly prefered the stimulus. Most neurons exhibited more modest changes in mean rate, with declines being almost as common as increases. For other areas (especially anaesthetized V1) it was the case that the vast majority of neurons increased their mean rate following stimulus onset. Nevertheless, for all datasets there was considerable overlap, before and after stimulus onset, in the distribution of mean rates. Mean-matching exploits this overlap. 
To apply mean-matching, for each neuron/condition we compute the mean and variance of the spike count in a sliding window (Fig. 4a). At each time, we plot the variances versus their respective means, with one data-point for every neuron/condition (Fig. 4b, gray and black points). The 'raw FF' is simply the slope of the regression relating the variance to the mean. The concern being addressed is that the raw FF might be lower following stimulus onset simply because rates are higher. Inspection reveals this to be unlikely: even for the range of mean counts that is shared across times, there are noticeably fewer high-variance points for the later times (compare -100 and $+100 \mathrm{~ms}$ ). Mean-matching formalizes this observation. We compute distributions of mean counts (gray marginal distributions) and take the greatest common distribution (black). At each time, individual points are randomly excluded until the actual distribution matches the common distribution. The mean-matched FF is based on the remaining (black) points.

All FFs reported in Figure 3 employed mean-matching. Very similar results were obtained for the raw FF (as in Fig. 4c). Thus, the decline is not somehow produced by meanmatching. Mean-matching simply controls for a potential artifact. Also note that, even following mean-matching, most of the analyzed neurons still showed stimulus-driven changes in mean rate (both increases and decreases). Only the overall distribution of firing rates is held constant. Before application to real data, mean-matching was applied to many simulated datasets, a few of which are shown in Supplementary Figure 2. The meanmatched FF successfully avoids artifacts related to changing mean rates over a wide range of circumstances, including low trial-counts, non-Poisson spiking, low mean rates, and large changes in mean rate.

\section{Further controls for the FF}

We employed a complementary method to further control for potential changes in spikingnoise statistics. We computed both the FF (for all neurons/conditions) and the square of the coefficient of variation of the inter-spike intervals $\left(\mathrm{CV}^{2}\right)$ using the method of 20 . While the FF declined following stimulus onset, the $\mathrm{CV}^{2}$ changed little (Supplementary Fig. 3). This is not consistent with a large change in spiking-process statistics (which should produce a large decline in both the $\mathrm{FF}$ and $\mathrm{CV}^{2}$ ) but is consistent with a decline in across-trial rate variability, a similar conclusion to that drawn in20. One can also control for rising mean rates (and resulting refractoriness) by focusing on conditions/neurons that individually exhibit little change in mean rate (e.g., null-directions and other non-responsive conditions). This necessitates that there exist a sufficient number of such cases to allow further analysis. Of the datasets in Figure 3, four (MT-plaids, PRR, PMd, and MT-speed) could be analyzed in this manner. In each case (Fig. 5), the FF decline is as clear for the non-responsive conditions $\left(\mathrm{p}<10^{-6}, \mathrm{p}<10^{-6}, \mathrm{p}<0.002, \mathrm{p}<10^{-4}\right)$ as it had been for the original analyses. This finding also addresses a second concern: low-rate neurons (which are preferentially preserved by mean-matching following stimulus onset) might tend to have low intrinsic FFs, resulting in an artifactual FF decline. However, such an artifact cannot affect the analysis in Figure 5: exactly the same neurons/conditions contribute at all times. Furthermore, the raw FF always showed the same decline as the mean-matched FF. In summary, the decline in the FF cannot be a simple consequence of refractoriness, changing spiking-noise statistics, or 
normalization. Rather it appears to reflect a widespread decline in the variance of the underlying firing rate.

\section{Variability declines even for 'non-responsive' conditions}

Analyses of non-responsive conditions (Fig. 2, Fig. 5) act a control, but also make a deeper point. The firing rate - averaged across trials - may change little, yet neurons are not truly non-responsive. It appears that underlying rates $d o$ change: from a variable range of rates before stimulus onset to a more consistent rate (with a similar mean) following stimulus onset. As in Figure 1c, it may be only the mean rate that changes little. The critical factor is not whether a neuron appears responsive from its mean, but whether the population as a whole is responsive. This is illustrated by the data from anaesthetized MT (Fig. 3, bottom). The onset of a static dot pattern evokes a weak and transient increase in mean rate at the population level, and a transient (though large) decline in the FF. In contrast, dot motion onset evokes a sustained increase in the mean population rate and a sustained FF decline. This FF decline is present and sustained even for those neurons where the mean rate is changing little (Fig. 5d). Thus, the FF decline depends on the stimulus effectively driving the population as a whole, but not on individual-neuron mean responsiveness. Consistent with this, the FF rises again fairly rapidly when the population ceases to respond following stimulus offset (Supplementary Fig. 4).

\section{A decline in correlated variability}

Does each neuron undergo its own decline in variability, or is the above effect related to variability that is shared? To assess this, our second approach exploits the covariance of simultaneous recordings, obtained via 96-electrode array recordings from two of the above areas: V1 and PMd. The measured covariance matrix (assessed across trials) reflects both shared firing-rate variability and 'private' spiking-process noise. It is thus necessary to decompose the measured covariance matrix into shared (network-level) and private components (Fig. 6a). A number of applicable methods exist, all of which exploit the assumption that shared variability should be relatively low dimensional (i.e., that neurons are tuned to a modest number of shared factors). Intuitively, if one can average across similarly tuned neurons to remove spiking noise, the underlying rate (and its variance) becomes more accessible. More precisely, if neurons are tuned for 8 shared factors, the covariance matrix describing the underlying rate will be of rank 8 . The measured covariance matrix should then be decomposed into a rank- 8 'network' covariance matrix, and a diagonal matrix capturing private spiking-process noise. Spiking-process variability is not necessarily physiologically cell-intrinsic $14,16,17$, but is weakly correlated between most neurons and thus 'private' for present purposes, especially given the long analysis windows being used. Of the applicable methods 22 , factor analysis 23 has the most appropriate noise model: different neurons may have different levels of private noise. This is essential, as Poisson spiking 'noise' scales with the mean rate. Supplementary Figure 5 further illustrates how FA separates network variability from private spiking noise.

We applied FA to four datasets: two V1 datasets from different animals and two PMd datasets collected from one animal on separate days. After isolating the network variances (blue diagonal in Fig. 6a) we computed the average network variance (across neurons/ 
conditions) before and after stimulus onset. The average variance was computed following a mean-matching procedure, just as was performed for the FF. Mean-matching aids interpretation by allowing direct comparison of the variance present for a given mean rate. It also insures that any contamination of network variability by private noise (inevitable for finite trial-counts) does not increase with rising rates. Non-mean-matched data are presented subsequently.

A decline in network variability was observed in all datasets (Fig. 6b,c,e,f). For V1, network variability was $66 \%$ and $40 \%$ lower following stimulus onset. For PMd, network variability was $53 \%$ and $45 \%$ lower. This decline was significantly greater for network variability than for private noise, in both absolute and relative terms (Fig. 6d,g and legend). Thus, the FF and FA analyses agree: stimulus onset drives a decline in firing-rate variability that is not simply due to a change in the regularity of spiking itself.

The decline in rate covariance is consistent with24 (from which one of the V1 datasets is drawn), in which correlations were lower overall after stimulus onset. However, the present result does not simply follow from that result. The mean pairwise correlation can decline even while overall covariance grows (e.g., if negative correlations become more common or if private spiking variability climbs). Interestingly, in three of the four cases, FA also indicates a small reduction in private noise. Limited data make it difficult to determine whether this indicates an actual change in spiking-process statistics or occurs because some network variability is incorrectly assigned to private noise. The latter can occur if the true dimensionality of the data is higher than the dimensionality assumed by the analysis (Supplementary Fig. 7a). This possibility is of minor concern: it would imply we are underestimating the network-variance decline. The converse (mis-assignment of a private change to a network change) is potentially concerning yet unlikely: for trial-shuffled data FA correctly assigns the variance change to private noise with only a small 'leak' into network variance (Supplementary Fig. 7b-e). Furthermore, the decline in network variability was the larger effect, both in absolute (Fig. 6b,c,e,f) and percentage (Fig. 6d,g) terms. In summary, there may or may not be modest changes in private spiking-process variability following stimulus onset. In either case, the measured decline in variability is principally a decline in network-level variability, shared among neurons.

As an aside, the shared variability being assessed is indeed variability in the firing rate across trials, not variability in precise spike patterns. Rerunning FA and FF analyses after 'jittering' each spike within a $20 \mathrm{~ms}$ window produced essentially identical results. This is unsurprising given the large (400 ms) analysis window.

\section{A qualification regarding the decline in variability}

For both the FF and FA, we compared identical distributions of mean rates before and after stimulus onset. Our central result must thus be stated carefully: for a given mean rate there is less variability around that mean following stimulus onset (e.g., the variance around a 10 spike/s mean might shrink from 15 to 8 spikes $^{2} / \mathrm{s}^{2}$ ). This will often imply that overall variability declines, but that can fail to occur when rates are initially very low. In the limit, when initial firing rates are zero, variability is zero and will necessarily rise when rates rise. For area PMd, overall firing rates change modestly following stimulus onset and overall 
variability does indeed decline. However, in anaesthetized V1 most neurons have baseline rates near zero, and thus near-zero variability. Overall variability is thus essentially guaranteed to rise following stimulus onset. This can be seen by plotting network-level rate variability versus mean rate (Fig. $6 \mathrm{~h}, \mathrm{i})$. In this analysis, spiking variability has been approximately factored out, leaving primarily underlying-rate variability. The effect of stimulus onset is essentially the same for both PMd and V1: the black curve lies well below the gray curve. For any given mean rate there is less variability around that rate after stimulus onset. However, for V1 mean rates are low before stimulus onset, necessitating low rate variance. Overall variability thus rises following stimulus onset (average variance rises from $77 \pm 5 \mathrm{SE}$ spikes $2 / \mathrm{s}^{2}$ to $190 \pm 8$ spikes $^{2} / \mathrm{s}^{2}$ ) even though it is lower for any given mean rate. For PMd, the overall change in mean rates is modest, and variance is lower after stimulus onset both for a given rate and in overall terms (average variance drops from $28 \pm 2$ spikes ${ }^{2} / \mathrm{s}^{2}$ to $16 \pm 1 \mathrm{spikes}^{2} / \mathrm{s}^{2}$ ). Thus, the central effect is a decline in the variability present around a given mean rate. This may or may not translate into an overall variance decline, depending on whether overall mean rates rise modestly or sharply. This qualification is restricted to the analysis of firing rate, whose variability must be zero when the mean is zero. By contrast, the membrane voltage shows considerable variability even when firing rates are near zero (Fig. 2).

\section{Single trial analysis of variability}

The 'neural response' is incompletely characterized by its mean. A fuller characterization is provided by also assessing firing-rate variance. Yet ideally one would directly observe the evolution of firing rates on individual trials, much as the membrane potential was directly observed on individual trials in Figure 2. Such visualization is likely important not only for the initially-high variance, but also for the variance that survives stimulus onset. The presence of remaining firing-rate variability after stimulus onset is underscored by its correlation with pre-stimulus variability2,6,8 (Supplementary note 1). The FA analysis (Fig. $6 \mathrm{~h}, \mathrm{i})$ also indicates that considerable firing-rate variability remains following stimulus onset. For PMd, such variability is behaviorally meaningful: it correlates with subsequent reaction time 9 and reach speed25. Estimating the evolution of single-trial firing rates is difficult or impossible based on single-neuron recordings, but becomes plausible for large-scale simultaneous recordings. To do so we employ apply an extension of FA termed 'Gaussianprocess factor analysis' (GPFA)26. GPFA projects the responses of many neurons into a low-dimensional space where each trial's 'neural trajectory' can be traced through time. Essentially, each axis captures the smoothed and averaged response of similarly-tuned neurons. More precisely, each neuron's underlying rate is a linear combination of the responses represented on each axis (much as for principal component analysis). A full characterization of single-trial variability across all conditions is beyond the scope of the present study. Still, the analyses below serve to illustrate the power, and in some cases the limitations, of viewing neural responses on a single-trial basis.

The collapse in variance in PMd is readily visualized via GPFA (Fig. 7a). The neural state is initially variable across trials, even though the monkey's external state - including fixation is tightly controlled. Following reach-target onset, the neural state becomes less variable and changes its center of mass (i.e., mean rates change). These features are particularly clear in 
the supplementary movies. Across both datasets and all 14 target locations, variability was always lower by the go cue than it had been pre-stimulus (for the two days, variance was an average of $27 \%$ and $37 \%$ smaller per dimension, relative to $100 \mathrm{~ms}$ pre-stimulus, $\mathrm{p}<10^{-6}$ and $\mathrm{p}<10^{-8}$; t-tests across 14 conditions). Variability was lower still by movement onset (36\% and $46 \%$ smaller than pre-stimulus variability; $\mathrm{p}<10^{-10}, \mathrm{p}<10^{-11}$ ).

Despite the above results, the principal goal of GPFA is not to further quantify variability and its decline (that is better accomplished by the FF and FA analyses). Rather, we would like to know how individual trials deviate from their mean. In particular, while most neural trajectories hewed close to their mean after target onset there were intriguing exceptions. For example, the red trace in Figure $7 \mathrm{~b}$ follows the usual trajectory up until the go cue (presumably indicating successful target detection and initial planning). Yet following the go cue the trajectory wanders before falling back on track. On this trial the monkey did indeed have an unusually long reaction time (629 ms; all others ranged from 240-375 ms). Other trials, for other target locations/days, contained similar outliers associated with long reaction times (Fig. 7c, Supplementary Fig. 8). Thus, across-trial averaging obscures not only the variance decline but also other interesting features, including events that may only have happened once.

We also applied GPFA to data from V1. Neural trajectories for one example condition (a $45^{\circ}$ drifting grating) are shown in Figure 8 . The initial overshoot corresponds to the onset transient; the looping trajectory is driven by the periodic stimulus. Across-trial variability is, if anything, more pronounced than in PMd. Individual-trials showed considerable variability in the stimulus-driven trajectory, and also in the trajectory before stimulus onset and after its offset. Of course, given a limited number of neurons, GPFA cannot provide noise-free estimates of single-trial firing rates. Yet much of the observed variability is real: shuffling the data to break neuron-neuron correlations greatly reduced variability relative to the original data (Fig. 8b,c). This was similarly true for every stimulus condition for all datasets tested. For the two V1 datasets, the original data had on average $81 \%$ and $89 \%$ more variance ( $\mathrm{p}<10^{-10}$ for both, t-test across conditions) than the shuffled data. The same was true for PMd (42\% and $43 \%$ more variance; $\mathrm{p}<10^{-15}$ for both). Thus, the variable individual-trial trajectories do indeed reflect trial-to-trial departures from the mean behavior. In particular, for $\mathrm{V} 1$ there is a surprising degree of variability in the initial state (Fig. 8c, blue), given the low average initial rates of most neurons. Because mean-matching cannot be applied to GPFA, total variability certainly did not decline following stimulus onset. Indeed it was higher, in agreement with Figure 6h. In summary, GPFA is in some ways less flexible than the FF or FA analyses (mean-matching cannot be applied, and the noise model is fixed across time). Still, the ability to reconstruct single-trial responses, and observe events that may only have occurred once, lends it a unique utility.

\section{Discussion}

Stimulus onset drives a widespread decline in the variability of the neural state. Consistent with prior reports, this effect is directly observable at the level of the membrane potential3,4,27. At the level of firing rates, the effect is not initially obvious. Much of the measured variability at the level of a single neuron is due to quasi-Poisson spiking statistics, 
whose variance rises with mean rate17. Nevertheless, appropriately-controlled metrics reveal that firing-rate variability declines sharply following stimulus onset. Specifically, for a given mean rate there is much less variability around that rate after stimulus onset. The ability to assess this effect in extracellular records was critical, as it allowed examination of datasets from many cortical areas, employing many stimuli, in both anesthetized and behaving animals. Stimulus onset might have been expected to impact neural variability differently depending on the area, stimulus, task, and behavioral state. Yet we consistently observed a decline in neural variability across all 14 datasets, from occipital to frontal cortex. This finding argues that a stimulus-driven decline in variability is a widespread feature of the cortical response.

The FF and FA methods both indicate that the central effect is a decline in the across-trial variability of the underlying firing rate, rather than a change in the spiking-statistics applied to that rate. This decline in firing-rate variability is likely a network property, for two reasons. First, the decline occurs even when the mean firing rate of a given neuron changes little (e.g., for non-preferred stimuli) provided that the network as a whole responds to the stimulus. Second, the variability in question is shared among neurons within an area, as revealed by FA. This finding, and the similar decline across areas, suggests that the relevant network could span much of cortex. Alternately, it may be that different areas exhibit the same effect simply because it is a common property of cortex. Distinguishing these possibilities will require large-scale simultaneous recordings from multiple areas.

Many prior studies have examined correlations between firing rate variability and behavior (e.g.,28). A few have related across-trial firing-rate variability with task difficulty,29 attention 21 or motor learning 30,31 . The present study is the first to fully document a simpler effect: the change in variability driven by the stimulus itself. That said, related effects can certainly be found in prior studies. This is true not only for the membrane potential3,4, but also for firing-rate variability. We previously reported (using methodology related to the FF) a variability decline in premotor cortex9. Variability also undergoes changes in motor cortex20,32. In retina, LGN and V1, the FF was lowest when rates were highest33. In a recent study of V421, the FF dropped after the stimulus entered the receptive field. There is also a small FF drop in previously-published MT data34. Variability declined throughout the trial for multiple areas during a probabilistic choice task35. Both cerebellar and FEF variability reach minima before movement 36,37 . A stimulus-driven decline in correlated activity5,24 was found for V1, consistent with a decline in (correlated) rate variability (also see38 for a similar effect r.e., LFP). Finally, there are reports39 of lower variability in driven versus spontaneous thalamic activity. In some of these studies variability changes were of passing interest, and controls for changing spiking-process statistics were not always applied (although see9,20). Yet our results argue that most of these effects likely reflect a decline in network-level firing-rate variance.

Mechanistically, the variance decline implies that cortical circuits become more stable when driven. Many network types can be stabilized by an input. Natural candidates are recurrent networks with attractor dynamics40. More broadly, a stimulus-driven decline in variability may be a general feature of large recurrent networks11,12. The widespread effects we observed may thus simply reflect the widespread presence of recurrent circuitry. These 
'network level' explanations are consistent with the shared nature of the firing-rate variability, and with our previous finding that a drop in PMd variability relates to reaction time9. Yet other data indicate a bottom-up source; in particular, shunting inhibition appears to be the source of the decline in V1 membrane-potential variability3. One hopes these two levels of explanation will be reconciled, although it is not yet clear how to do so.

The functional interpretation of converging rates likely varies by area. For PMd, the decline may relate to motor preparation9,10. In area V4, it may relate to attention21. Often it is tempting to relate the variability decline to cognitive-level phenomena: stimulus onset is a natural candidate to focus not only attention and planning, but other forms of mental processing as well. On the other hand, the decline was observed even under anesthesia (all V1 datasets and two of the MT datasets) consistent with lower-level explanations3. See Supplementary note 2 for further discussion of the possible origins - local versus cognitive of the initial variability. The anesthetized data additionally rule out effects related to microsaccades or other unobserved changes in behavior. Finally, effects in the anaesthetized data appear unrelated to long-timescale drift of anesthesia: the decline is undiminished when computed more locally in time (Supplementary note 3 ).

Despite being widespread, the changes in variability are unlikely to constitute a 'coding channel'. It is unlikely that the brain could measure or interpret variability that is present only across trials. It is the experimenter who, when interpreting the recorded responses, needs to know when variability is substantial and how it is changing. Most basically, we need to know when the mean is representative. More intriguingly, different classes of dynamics - single-attractor, multi-attractor, integrator - can have different variability 'signatures' (A.K. Churchland, R Kiani \& M.N. Shadlen, Soc. Neurosci. Abstr., 2006). Variability measurements may thus allow hypotheses regarding circuit-level dynamics to be tested at the level of the single neuron. More generally, while the mean and the variance are indispensable metrics, one also wishes to observe the individual-trial responses themselves. GPFA and related methods can approximately reconstruct individual-trial neural trajectories from simultaneous extracellular recordings41, and thus have the potential to uncover telling features of the neural response that are normally lost to averaging.

\section{Methods}

\section{Datasets}

All methods were approved in advance by the relevant institutional animal care and use committees. Data were collected from seven cortical areas of the macaque monkey and one cortical area of the cat by members of nine laboratories. For most datasets, methods have been described previously4,9,24,42,43,44,45,46,47. Briefly, for the intracellularly-recorded V1 datasets, stimuli were $100 \%$ contrast sine-wave gratings presented at different orientations. For the extracellularly-recorded V1 dataset, stimuli were $100 \%$ contrast sinewave gratings $\left(6.25 \mathrm{~Hz}, 1.3\right.$ cycles $\left.^{\circ}\right)$ drifting in one of 12 directions. For V4, stimuli were one or two oriented bars situated in the neuron's receptive field. For some conditions, similar bars appeared in the opposite hemifield. For the first MT dataset, stimuli were square-wave gratings superimposed to produce a plaid. These patterns contained a small amount of variable-contrast texture, which was identical for all trials of a given type within a 
recording session (making it sensible to compute the response variance across those trials). For the second MT dataset, stimuli were $0 \%$-coherence random dots. These had exactly the same dot pattern for repeated trials within a session. For LIP, stimuli were two colored saccade targets, between which the monkey chose. Analysis was further segregated by the eventual choice and the estimated value of that choice to the monkey. E.g., one of the conditions corresponded to rightwards saccades to a red target of high reward probability. For PRR, stimuli were reach/saccade targets (modality instructed by color) both within and outside the neuron's receptive field. For PMd, stimuli were reach targets at one of 2 distances and 7 directions. For OFC, the task was similar to that used for LIP, but analysis was locked to reward delivery (after the saccade). Analysis was segregated based on the features that OFC neurons seemed most sensitive to: choice, whether that choice repeated the last choice, and whether reward was delivered. E.g., one of the conditions corresponded to rewarded saccades to a red target when that target was chosen last time. For the third and fourth MT datasets, stimuli were random dot patterns. For 'MT-direction/area', stimulus direction and dot-patch area varied across conditions. For the 'MT-speed' dataset, stimuli were presented at different speeds $(1-128 \% \mathrm{~s})$ in the preferred and null directions. For these two datasets, the exact location of the individual dots within the aperture was different on each trial. This presumably did inflate the FF by injecting additional across-trial variability. However, this appears to be a modest concern: robust FF declines were still observed. These two datasets were also unusual in that the stimulus (the dot patch) appeared at the moment the trial began (and data collection commenced). Motion onset occurred $256 \mathrm{~ms}$ later.

Extracellular V1, MT-direction/area, and MT-speed datasets were recorded from opioidanaesthetized monkeys (macaca fascicularis or macaca nemestrina). Intracellular V1 recordings were made from anaesthetized cats. All others were made from awake monkeys (macaca mulatta) performing a task with controlled fixation. For the V4 dataset, the task was simply to fixate while stimuli were presented. For PMd, the task required a reach. For PRR the task required a reach or a saccade. For the remaining datasets the task required a saccade indicating a choice or perceptual discrimination. Tasks involved a delay between stimulus onset and when a response was allowed, with one exception. For the MT-dots dataset, the monkey was allowed to respond as soon as he had made the discrimination. Analysis was thus limited to reaction times longer than $250 \mathrm{~ms}$ to allow a sufficient analysis window. All tasks involved a period of enforced fixation prior to stimulus onset. For LIP this was not true for all trials, and analysis was thus restricted to those that did. Fixation enforcement makes it unlikely that changes in variability are indirectly due to saccades. Additionally, datasets recorded from anaesthetized/paralyzed animals showed the same changes in variability. Finally, it is unlikely that changes in the FF, with a latency of $\sim 60 \mathrm{~ms}$, could be indirectly due to saccades, which would incur a latency of 80-200 ms48 plus an additional visual delay of $40-80 \mathrm{~ms}$ before firing rates could be affected. For all analyses, each trial's data were aligned on stimulus onset.

Most datasets were recorded across multiple days (and for LIP and MT, more than one monkey) using single electrodes. Exceptions were the V1 and PMd datasets. (The following applies to the FF analysis. See below regarding FA and GPFA.) The V1 data (dataset 565L) were recorded in one day from a 96 channel electrode array (Cyberkinetics). Only single- 
unit isolations (44) were analyzed. The PMd dataset was recorded using a similar array.

Data were pooled across 5 days to increase trial and neuron count (although many units were likely recorded repeatedly). Single-neuron counts for the datasets used for the FF analysis were: 44 (V1), 49 (V4), 314 (MT plaids), 82 (MT dots), 43 (LIP), 175 (PRR), 73 (PMd), 116 (OFC), 47 (MT-direction/area), and 57 (MT-speed). The mean number of trials/ condition was 100 (V1), 24 (V4), 15 (MT plaids), 88 (MT dots), 35 (LIP), 10 (PRR), 31 (PMd), 106 (OFC), 125 (MT-direction/ area), and 14 (MT-speed). The total numbers of 'neuron-trials' (number of neurons times the number of trials per neuron) were 52,800 (V1), 7063 (V4), 106,007 (MT plaids), 7179 (MT dots), 8357 (LIP), 14,160 (PRR), 35,953 (PMd), 49,389 (OFC), 61,343 (MT-direction/area) and 12,797 (MT-speed).

FA requires simultaneously recorded data (e.g., array-based recordings from a single day). Simulations similar to that in Supplementary Figure 5 convinced us that the V1 and PMd datasets used for the FF analyses had neuron/trial-counts barely sufficient to provide FA with sufficient statistical power. To increase statistical power we included multi-unit isolations in the analysis. We used the two PMd recording days with the most (> 50) trials per condition (G20040123 \& G20040122). For V1, we employed two datasets (567R and 106R). Both used the same stimuli as the dataset used for the FF (described above) but had higher unit/trial counts. The first (567R) had only 20 single-unit isolations, but many multiunit isolations (102) and more trials/condition (200 rather than 100) relative to the dataset used for the FF analysis. The second dataset (106R) had a very large (172) number of primarily multi-unit isolations, and even more trials/condition (400). These datasets were thus well-suited to the FA analysis. Both these datasets exhibited very clear FF declines (Supplementary Fig. 6). However, the decision was made to keep the original FF analysis (Fig. 3) restricted to the single-unit-only dataset. While the FF can be reliably computed for multi-unit recordings (the sum of independent Poisson-distributed counts is Poissondistributed), the mean-matching procedure is not guaranteed to work.

\section{Fano factor}

The FF was computed using matlab (The Mathworks) code available at (http:// www.stanford.edu/ shenoy/GroupCodePacks.htm). Data for each neuron and condition are initially treated separately. A condition corresponds to multiple trials of the same stimulus. Spike counts were computed in a $50 \mathrm{~ms}$ sliding window moving in $10 \mathrm{~ms}$ steps. We then computed the variance (across trials) and mean of the spike count. Thus, the data for neuron $_{1}$, condition $_{1}$, time $_{1}$ were reduced to two numbers: the variance and mean of the spike count in a window centered on that time. For each time, a scatterplot of the variance versus the mean was compiled, with one point per neuron/condition (typically a few thousand points). The 'raw' FF was the slope of the regression relating the variance to the mean. This regression was weighted according to the estimated sampling error for the variance (computed based on the number of trials and the mean rate, and assuming Poisson statistics). The observed effects are not due to the use of a short $(50 \mathrm{~ms})$ window: effect magnitudes were generally larger with larger windows, at the cost of blurred timecourses.

To compute the 'mean-matched Fano factor', we first computed the distribution of mean counts (one datum per neuron/condition) for each time being analyzed (grey distributions; 
Fig. 4b). We then computed the greatest common distribution present at all times (black distribution). Each bin of this common distribution had a height equal to the smallest value for that same bin across all distributions at all times. For each time, we then matched the analyzed distribution of mean rates to this common distribution. This was accomplished by discarding, from each bin, randomly chosen datapoints (each corresponding to the variance/ mean pair for one neuron/condition) until the height of that bin matched that of the common distribution. The FF was then computed. This procedure was repeated 50 times with different random seeds, with a different subset of the data preserved on each repetition, and thus a slightly different resulting FF. The reported FF is the mean of these individual FFs.

This mean-matching method discards less data than might be expected, and far less than if we restrict analysis to individual neurons whose rates were not changing for particular conditions (as in Fig. 5). Mean matching preserved 49\% (V1), 31\% (V4), 71\% (MT plaids), $32 \%$ (MT dots), 48\% (LIP), 84\% (PRR), 75\% (PMd), 86\% (OFC), 46\% (MT-direction/area) and $70 \%$ (MT-speed) of the 'neuron-conditions'. Prior studies using the FF have occasionally presented controls involving informal mean-matching: drawing attention to different values of the FF over a similar range of rates $9,21,33$. The current method removes the need for such additional controls. Mean-matching also does more than validate the basic effect; it prevents artifacts from corrupting the timecourse (Supplementary Fig. 2).

The statistical significance of the FF decline was assessed by comparing the FF $100 \mathrm{~ms}$ before stimulus onset and $200 \mathrm{~ms}$ after (for the MT-direction/area and MT-speed datasets, where the FF underwent two declines, we compared the time of motion onset with $200 \mathrm{~ms}$ after). Significance was computed based on sampling distributions estimated from the $95 \%$ confidence intervals yielded by the regression used to compute the FF. The decline was statistically significant in all cases: $\mathrm{p}<0.001(\mathrm{~V} 1), \mathrm{p}<10^{-5}(\mathrm{~V} 4), \mathrm{p}<10^{-10}$ (MT plaids), $\mathrm{p}<0.01$ (MT dots), $\mathrm{p}<0.005$ (LIP), $\mathrm{p}<0.002$ (PRR), $\mathrm{p}<10^{-10}$ (PMd), $\mathrm{p}<0.02$ (OFC), $\mathrm{p}<10^{-14}$ (MTdirection/area), and $\mathrm{p}<10^{-7}$ (MT-speed).

\section{Factor analysis}

FA was applied to spike counts in a $400 \mathrm{~ms}$ window, which either ended at stimulus onset ('Pre-stimulus') or began $100 \mathrm{~ms}$ after stimulus onset ('Stimulus'). For the $2^{\text {nd }} \mathrm{V} 1$ dataset (Fig. 6c), stimulus duration was only $400 \mathrm{~ms}$, so we used a shorter $(350 \mathrm{~ms})$ window that began $50 \mathrm{~ms}$ after stimulus onset. A data matrix, $\mathrm{D}$, was compiled, with $\mathrm{D}_{i j}$ being the spike count for the $i^{\text {th }}$ trial and the $j^{\text {th }}$ neuron. D included only neurons with rates $>=1$ spike/s for both periods. FA was then applied to this matrix (neurons are variables, trials are observations). FA was performed separately on the D matrices compiled before and after stimulus onset. FA was performed separately for each condition. Subsequent results were averaged. We used code written by Z. Ghahramani22 to fit the FA parameters using expectation-maximization. Essentially identical results were obtained using matlab's 'factoran'. FA requires an estimate of the dimensionality of the latent space capturing shared variability. To estimate this, we exploited the fact that recordings were made for multiple stimulus conditions (14 for PMd, 12 and 8 for the two V1 datasets). We computed the mean (across time and trials) response for each condition. We also included the pre-stimulus period as a condition. We then applied PCA (to the $c \times n$ matrix) and asked how many 
dimensions were necessary to account for $95 \%$ of the across-condition response variance (PCA is acceptable because it is being applied to trial-averaged data). For PMd and V1, 5 and 4 dimensions were required. These were then the latent dimensionalities assumed by FA. These numbers likely underestimate of the true dimensionality of the network response across conditions (our stimuli spanned only part of the space of all stimuli). This underestimate is acceptable: it implies that FA will underestimate the drop in the network variance, making our current interpretation conservative (Supplementary Fig. 7). Finally, similar results were obtained across a range of reasonable latent dimensionalities (2-8).

We used FA to estimate the private and shared component of each neuron's variance (that is, we computed but did not analyze the full network covariance matrix). This kept each variance associated with a mean rate, and allowed us to apply the mean-matching procedure used for the FF. Mean-matching aids interpretation because 1) even when rates are rising dramatically, we can ask whether rate variance is changing for a given mean rate 2) there is a clear expectation that the estimated private noise should change little (assuming effects are not due to private noise), and 3) there is less concern that a large increase in private noise (which will occur with rising mean rates) might contaminate the network variance estimate. The percentage of neuron/conditions preserved following mean-matching was $31 \%$ and $48 \%$ (two V1 datasets) and 74\% and 79\% (two PMd datasets).

The 'estimated variances' plotted in Figure 6b,c,e,f are the mean network (shared) variances, averaged across neurons/conditions, after mean-matching. Significance was tested via t-test ( $n=$ number of conditions).

\section{Gaussian-process factor analysis}

GPFA26 was used to extract temporally-smooth single-trial neural trajectories. Spike counts were taken in non-overlapping $20 \mathrm{~ms}$ bins, then square-rooted. The square-root transform 49 allows GPFA (which assumes stationary private noise for each neuron) to handle what would otherwise be a time-varying scaling of private noise with changing rates. Unlike FA, GPFA was applied simultaneously to data from all timepoints. It was critical that FA was applied separately before and after stimulus onset, to allow it to capture any changes in private noise. For GPFA we require a visualization in which the same latent space captures the neural trajectory across time. Using all timepoints also increases the quantity of analyzable data. The cost is that GPFA, unlike the FA analysis, cannot account for changes in private noise with time. However, such changes are likely modest (Fig. 6b,c,e,f) and are not the focus of the GPFA analysis (which seeks to track changes in network state, not private noise).

Figure 7 and Figure 8 plot 2-dimensional projections of the higher-dimensional trajectories found by GPFA. For PMd, subsequent analysis quantified ellipsoid volumes at different times. This analysis employed the higher-dimensional latent space. The dimensionality of that space was estimated using cross-validation. This is reasonable for GPFA, relative to FA (where we did not use cross-validation), because of the increase in datapoints gained by including different times. Across the 14 reach targets, each analyzed separately, the latent dimensionality ranged from 8 to 12 . It is expected that this dimensionality is higher than that for FA, as the latter needed only to account for variability at a single time, while GPFA must 
account for variability across both trials and time. GPFA produced similar results when we used a dimensionality smaller (6) or higher (15) than that chosen by cross validation. Because dimensionality impacts the covariance volume, we report that volume per dimension (i.e., volume ${ }^{\wedge}(1 / k)$ where $k$ is the latent dimensionality; $k$ was always the same pre- and post-target).

For PMd, the 2D projections in Figure 7 were chosen to preserve the relative perdimensional volumes of the 8-12 dimensional covariance ellipses. For V1, we simply plotted the top two dimensions returned by GPFA, which maximizes the captured variability (analogous to the first two principal components).

\section{Supplementary Material}

Refer to Web version on PubMed Central for supplementary material.

\section{Acknowledgements}

This work was supported by a Helen Hay Whitney postdoctoral fellowship (MMC), Burroughs Welcome Fund Career Awards in the Biomedical Sciences (MMC, KVS), NIH Director's Pioneer Award 1DP1OD006409 (KVS), Gatsby Charitable Foundation (MS, BMY), National Institute of Health NINDS Collaborative Research in Computational Neuroscience Grant 5-R01-NS054283-02 (KVS, MS), the Michael Flynn Stanford Graduate Fellowship and NIH-NINDS-CRCNS-R01 (JPC), the Howard Hughes Medical Institute and NIH grant EY05603 (WTN, LPS, MRC, GSC), an HHMI predoctoral fellowship (MRC), NIH grant NIH EY014924 (TM, KMA), Sloan Foundation (TM, KVS), Pew Charitable Trust (TM), NIH EY015958 and EY018894 (MAS), NIH EY02017 and EY04440 (JAM), NIH EY016774 (AK), NIH 1 EY13138-01 (DCB, AMC, PH, BBS), NIH EY019288 (NJP), the Pew Charitable Trust (NJP), EY04726 (DF), NDSEG Fellowships (BMY, GS), NSF Graduate Research Fellowships (BMY, GS), the Christopher and Dana Reeve Foundation (KVS, SIR), and the following awards to KVS:, Stanford Center for Integrated Systems, National Science Foundation Center for Neuromorphic Systems Engineering at Caltech, Office of Naval Research, and Whitaker Foundation. We thank Stephen G. Lisberger, in whose laboratory two of the datasets (MT-direction/area and MT-speed) were collected.

\section{References}

1. Briggman KL, Abarbanel HD, Kristan WB Jr. Optical imaging of neuronal populations during decision-making. Science. 2005; 307:896-901. [PubMed: 15705844]

2. Arieli A, Sterkin A, Grinvald A, Aertsen A. Dynamics of ongoing activity: explanation of the large variability in evoked cortical responses. Science. 1996; 273:1868-1871. [PubMed: 8791593]

3. Monier C, Chavane F, Baudot P, Graham LJ, Fregnac Y. Orientation and direction selectivity of synaptic inputs in visual cortical neurons: a diversity of combinations produces spike tuning. Neuron. 2003; 37:663-680. [PubMed: 12597863]

4. Finn IM, Priebe NJ, Ferster D. The emergence of contrast-invariant orientation tuning in simple cells of cat visual cortex. Neuron. 2007; 54:137-152. [PubMed: 17408583]

5. Kohn A, Zandvakili A, Smith MA. Correlations and brain states: from electrophysiology to functional imaging. Curr Opin Neurobiol. 2009; 19:434-438. doi:S0959-4388(09)00063-4 [pii] 10.1016/j.conb.2009.06.007. [PubMed: 19608406]

6. Azouz R, Gray CM. Cellular mechanisms contributing to response variability of cortical neurons in vivo. J Neurosci. 1999; 19:2209-2223. [PubMed: 10066274]

7. Fiser J, Chiu C, Weliky M. Small modulation of ongoing cortical dynamics by sensory input during natural vision. Nature. 2004; 431:573-578. [PubMed: 15457262]

8. Kisley MA, Gerstein GL. Trial-to-trial variability and state-dependent modulation of auditoryevoked responses in cortex. J Neurosci. 1999; 19:10451-10460. [PubMed: 10575042]

9. Churchland MM, Yu BM, Ryu SI, Santhanam G, Shenoy KV. Neural variability in premotor cortex provides a signature of motor preparation. J Neurosci. 2006; 26:3697-3712. [PubMed: 16597724] 
10. Rickert J, Riehle A, Aertsen A, Rotter S, Nawrot MP. Dynamic encoding of movement direction in motor cortical neurons. J Neurosci. 2009; 29:13870-13882. doi:29/44/13870 [pii] 10.1523/ JNEUROSCI.5441-08.2009. [PubMed: 19889998]

11. Sussillo D, Abbott LF. Generating coherent patterns of activity from chaotic neural networks. Neuron. 2009; 63:544-557. [PubMed: 19709635]

12. Abbott, LF.; Rajan, K.; Sompolinsky, H. Neuronal Variability and its Functional Significance. Ding, M.; Glanzman, D., editors. (in press)

13. Poulet JF, Petersen CC. Internal brain state regulates membrane potential synchrony in barrel cortex of behaving mice. Nature. 2008; 454:881-885. [PubMed: 18633351]

14. Shadlen MN, Newsome WT. The variable discharge of cortical neurons: implications for connectivity, computation, and information coding. J Neurosci. 1998; 18:3870-3896. [PubMed: 9570816]

15. van Vreeswijk C, Sompolinsky H. Chaos in neuronal networks with balanced excitatory and inhibitory activity. Science. 1996; 274:1724-1726. [PubMed: 8939866]

16. Mainen ZF, Sejnowski TJ. Reliability of spike timing in neocortical neurons. Science. 1995; 268:1503-1506. [PubMed: 7770778]

17. Carandini M. Amplification of trial-to-trial response variability by neurons in visual cortex. PLoS Biol. 2004; 2:E264. [PubMed: 15328535]

18. Tolhurst DJ, Movshon JA, Dean AF. The statistical reliability of signals in single neurons in cat and monkey visual cortex. Vision Res. 1983; 23:775-785. [PubMed: 6623937]

19. Gur M, Beylin A, Snodderly DM. Response variability of neurons in primary visual cortex (V1) of alert monkeys. J Neurosci. 1997; 17:2914-2920. [PubMed: 9092612]

20. Nawrot MP, et al. Measurement of variability dynamics in cortical spike trains. J Neurosci Methods. 2008; 169:374-390. [PubMed: 18155774]

21. Mitchell JF, Sundberg KA, Reynolds JH. Differential attention-dependent response modulation across cell classes in macaque visual area V4. Neuron. 2007; 55:131-141. [PubMed: 17610822]

22. Roweis S, Ghahramani Z. A unifying review of linear gaussian models. Neural Comput. 1999; 11:305-345. [PubMed: 9950734]

23. Everitt, BS. An introduction to latent variable models. Chapman \& Hall; 1984.

24. Smith MA, Kohn A. Spatial and temporal scales of neuronal correlation in primary visual cortex. J. Neurosci. 2008; 28:12591-12603. [PubMed: 19036953]

25. Churchland MM, Afshar A, Shenoy KV. A central source of movement variability. Neuron. 2006; 52:1085-1096. [PubMed: 17178410]

26. Yu BM, et al. Gaussian-process factor analysis for low-dimensional single-trial analysis of neural population activity. J Neurophysiol. 2009; 102:614-635. [PubMed: 19357332]

27. Monier C, Fournier J, Fregnac Y. In vitro and in vivo measures of evoked xcitatory and inhibitory conductance dynamics in sensory cortices. J Neurosci Methods. 2008; 169:323-365. doi:S0165-0270(07)00554-7 [pii] 0.1016/j.jneumeth.2007.11.008. [PubMed: 18215425]

28. Britten KH, Newsome WT, Shadlen MN, Celebrini S, Movshon JA. A relationship between behavioral choice and the visual responses of neurons in macaque MT. Vis Neurosci. 1996; 13:87100. [PubMed: 8730992]

29. Horwitz GD, Newsome WT. Target selection for saccadic eye movements: prelude activity in the superior colliculus during a direction-discrimination task. J Neurophysiol. 2001; 86:2543-2558. [PubMed: 11698541]

30. Mandelblat-Cerf Y, Paz R, Vaadia E. Trial-to-trial variability of single cells in motor cortices is dynamically modified during visuomotor adaptation. J Neurosci. 2009; 29:15053-15062. doi: 29/48/15053 [pii] 10.1523/JNEUROSCI.3011-09.2009. [PubMed: 19955356]

31. Kao MH, Doupe AJ, Brainard MS. Contributions of an avian basal ganglia-forebrain circuit to real-time modulation of song. Nature. 2005; 433:638-643. [PubMed: 15703748]

32. Oram MW, Hatsopoulos NG, Richmond BJ, Donoghue JP. Excess synchrony in motor cortical neurons provides redundant direction information with that from coarse temporal measures. $\mathrm{J}$ Neurophysiol. 2001; 86:1700-1716. [PubMed: 11600633] 
33. Kara P, Reinagel P, Reid RC. Low response variability in simultaneously recorded retinal, thalamic, and cortical neurons. Neuron. 2000; 27:635-646. [PubMed: 11055444]

34. Osborne LC, Bialek W, Lisberger SG. Time course of information about motion direction in visual area MT of macaque monkeys. J Neurosci. 2004; 24:3210-3222. [PubMed: 15056700]

35. Lee, D.; Seo, H. Neuronal variability and its functional significance. Glanzman, D.; Ding, M., editors. Oxford Press; in press

36. Fortier PA, Smith AM, Kalaska JF. Comparison of cerebellar and motor cortex activity during reaching: directional tuning and response variability. J Neurophysiol. 1993; 69:1136-1149. [PubMed: 8492153]

37. Cohen JY, et al. Difficulty of visual search modulates neuronal interactions and response variability in the frontal eye field. J Neurophysiol. 2007; 98:2580-2587. [PubMed: 17855586]

38. Nauhaus I, Busse L, Carandini M, Ringach DL. Stimulus contrast modulates functional connectivity in visual cortex. Nat Neurosci. 2009; 12:70-76. [PubMed: 19029885]

39. Werner G, Mountcastle VB. The Variability Of Central Neural Activity In A Sensory System, And Its Implications For The Central Reflection Of Sensory Events. J Neurophysiol. 1963; 26:958977. [PubMed: 14084169]

40. Wang XJ. Decision making in recurrent neuronal circuits. Neuron. 2008; 60:215-234. doi:S0896-6273(08)00836-2 [pii] 10.1016/j.neuron.2008.09.034. [PubMed: 18957215]

41. Churchland MM, Yu BM, Sahani M, Shenoy KV. Techniques for extracting single-trial activity patterns from large-scale neural recordings. Curr Opin Neurobiol. 2007; 17:609-618. [PubMed: 18093826]

42. Sugrue LP, Corrado GS, Newsome WT. Matching behavior and the representation of value in the parietal cortex. Science. 2004; 304:1782-1787. [PubMed: 15205529]

43. Cohen MR, Newsome WT. Context-dependent changes in functional circuitry in visual area MT. Neuron. (in press).

44. Armstrong KM, Fitzgerald JK, Moore T. Changes in visual receptive fields with microstimulation of frontal cortex. Neuron. 2006; 50:791-798. [PubMed: 16731516]

45. Armstrong KM, Moore T. Rapid enhancement of visual cortical response discriminability by microstimulation of the frontal eye field. Proc Natl Acad Sci U S A. 2007; 104:9499-9504. [PubMed: 17517599]

46. Chang SW, Dickinson AR, Snyder LH. Limb-specific representation for reaching in the posterior parietal cortex. J Neurosci. 2008; 28:6128-6140. [PubMed: 18550755]

47. Priebe NJ, Churchland MM, Lisberger SG. Constraints on the source of short-term motion adaptation in macaque area MT. I. the role of input and intrinsic mechanisms. J Neurophysiol. 2002; 88:354-369. [PubMed: 12091560]

48. Boch R, Fischer B. Saccadic reaction times and activation of the prelunate cortex: parallel observations in trained rhesus monkeys. Exp Brain Res. 1983; 50:201-210. [PubMed: 6641855]

49. Kihlberg JK, Herson JH, Schotz WE. Square root transformation revisited. Appl. Statist. 1972; 21:76-81. 
a
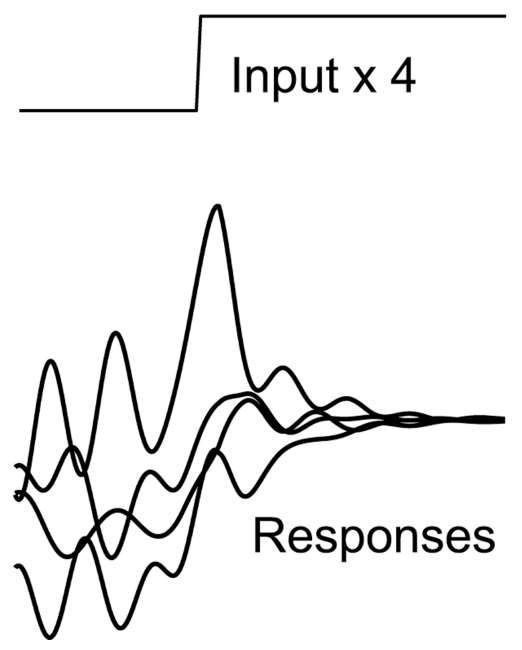

b
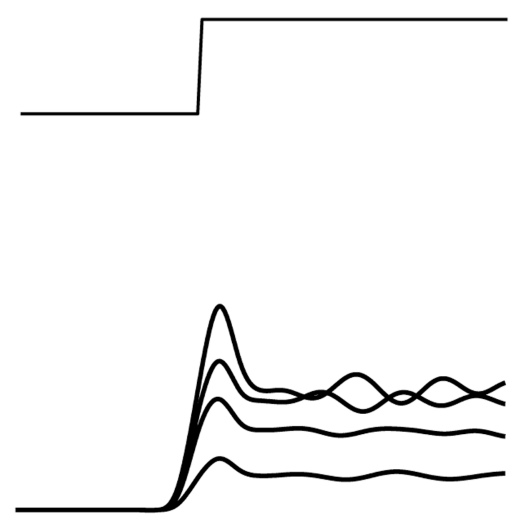

C

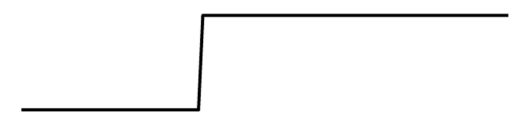

M

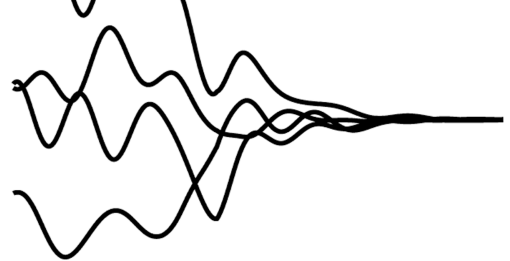

Figure 1.

Schematic illustration of possible types of across-trial firing rate variability. In each panel, we suppose that the same stimulus is delivered four times (four trials) yielding four different responses. Panels $a$ and $b$ were constructed to have the same mean response across the four trials. $\boldsymbol{a}$. Stimulus-driven decline in variability. $\boldsymbol{b}$. Stimulus-driven rise in variability. $\boldsymbol{c}$. Stimulus-driven decline in variability with little change in mean rate. 




b
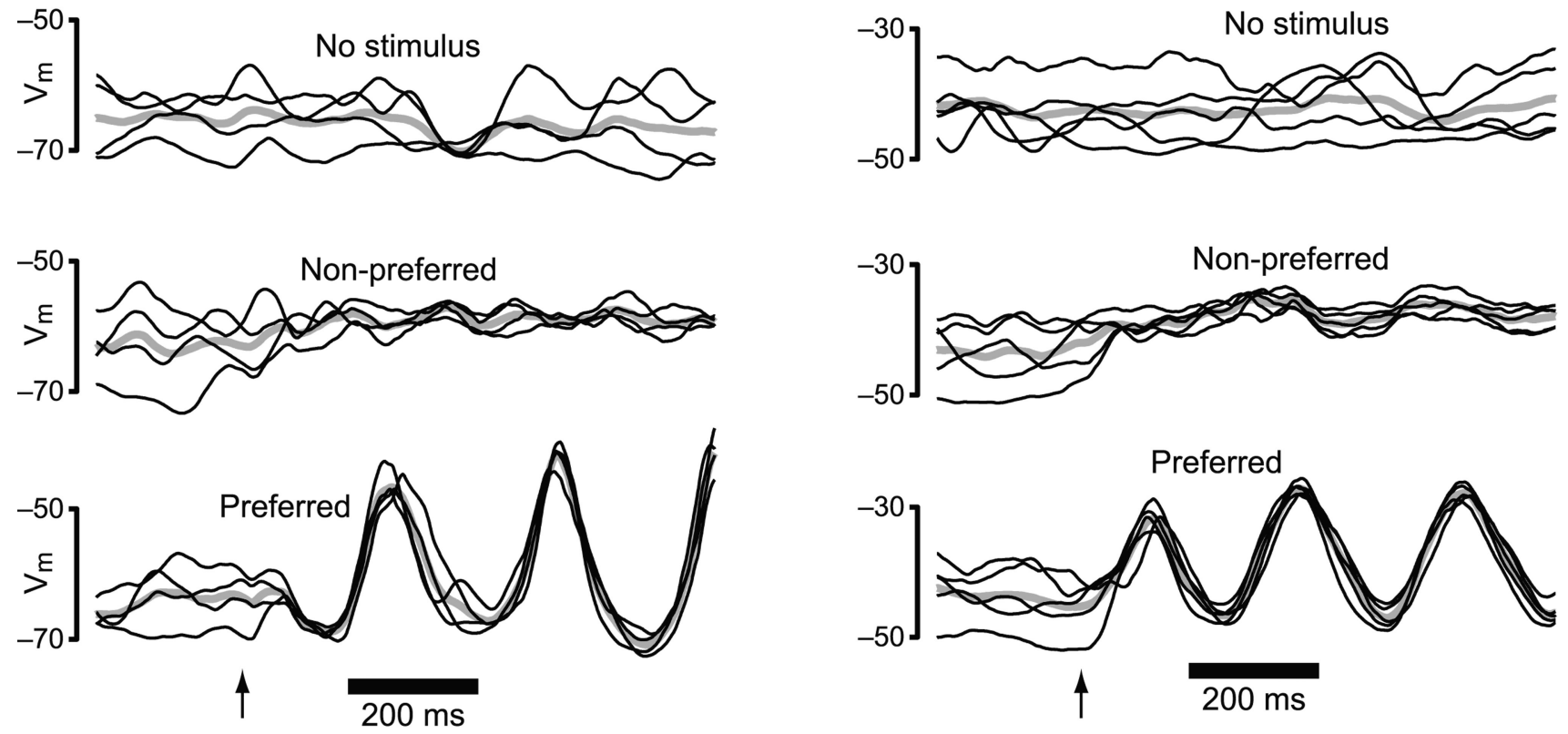

C


Figure 2.

Analysis of intracellularly-recorded membrane potential from cat V1. Stimuli were drifting sine-wave gratings presented at different orientations / frequencies. Analysis employed a 50 ms sliding window (box filter) to match the $50 \mathrm{~ms}$ window used for the FF analysis. Similar results were obtained with a shorter $(5 \mathrm{~ms})$ or longer $(100 \mathrm{~ms})$ window. $\boldsymbol{a}$. Data from one example neuron. $\mathrm{V}_{\mathrm{m}}$ for individual trials (black) is plotted on top of the mean (gray). Data are shown when no stimulus was delivered, for a non-preferred stimulus, and for a preferred stimulus. The arrow marks stimulus onset. $\boldsymbol{b}$. Similar plot for a second example neuron. $\boldsymbol{c}$. 
The mean and variance of $\mathrm{V}_{\mathrm{m}}$ across all 52 neurons and all stimuli. Flanking traces give SEMs. 

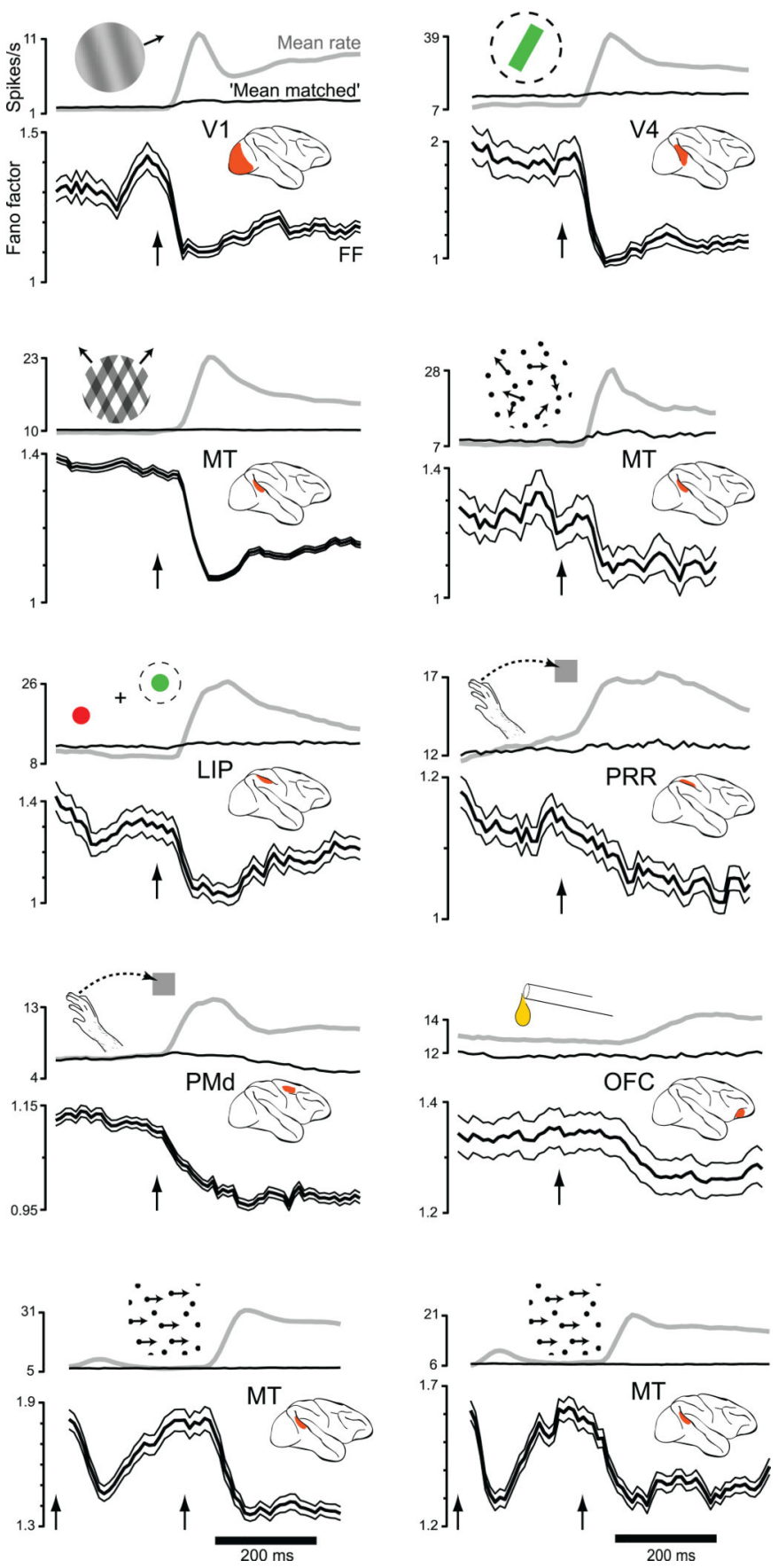

Figure 3.

Changes in firing-rate variance for eight datasets (one per panel). Insets indicate stimulus type. Data are aligned on stimulus onset (arrow). For the two bottom panels (MT-area/ direction and MT-speed) the dot pattern appeared at time zero (first arrow) and began moving at the second arrow. The mean rate (gray) and the FF (black with flanking SEs) were computed using a $50 \mathrm{~ms}$ sliding window. For OFC, where response amplitudes were small, a $100 \mathrm{~ms}$ window was used to gain statistical power. Analysis included all conditions, including non-preferred. The FF was computed after mean-matching (Fig. 4). The resulting 
stabilized means are shown in black. The mean number of trials/condition was 100 (V1), 24 (V4), 15 (MT plaids), 88 (MT dots), 35 (LIP), 10 (PRR), 31 (PMd), 106 (OFC), 125 (MTdirection/area), and 14 (MT-speed). 

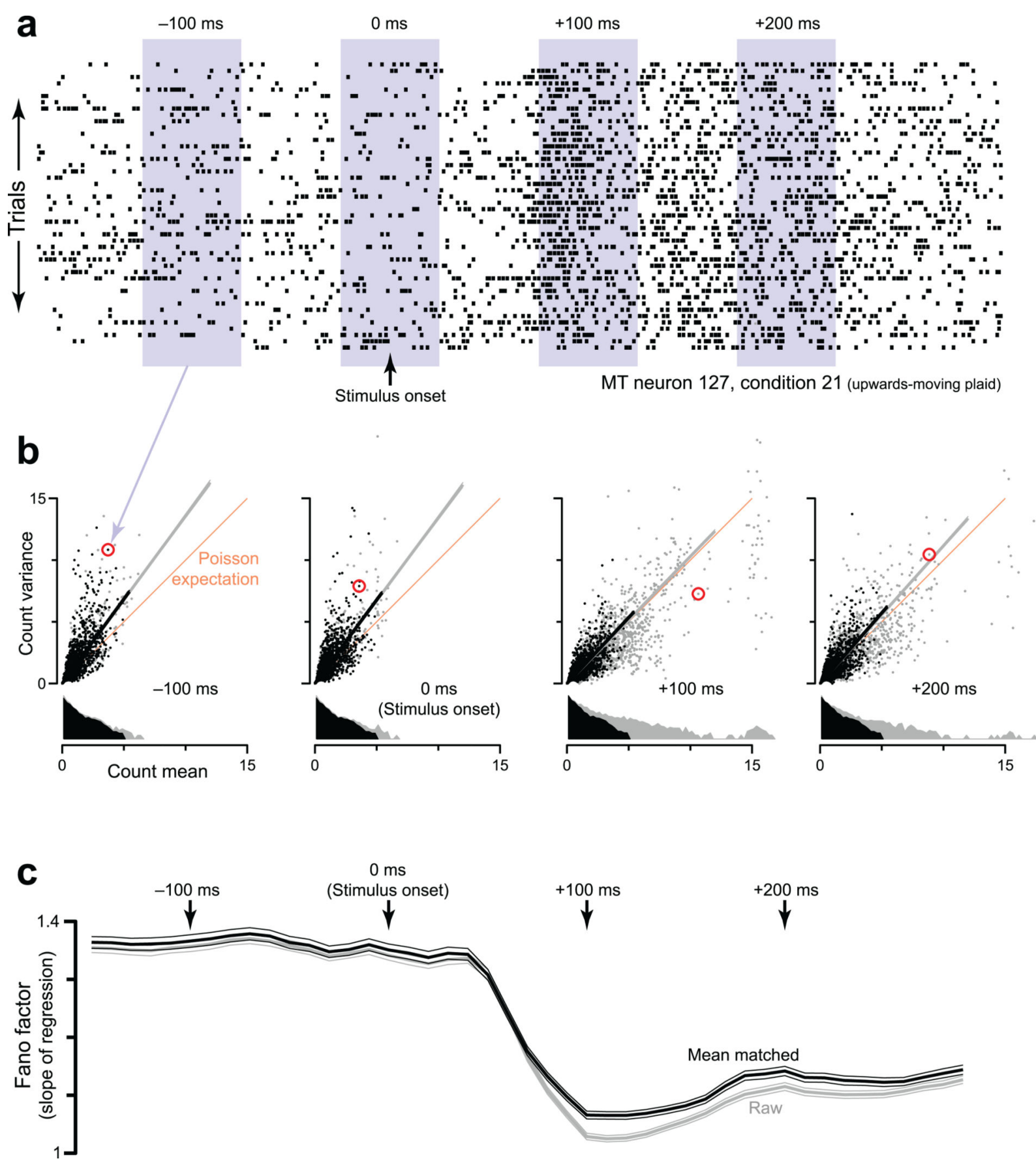

Figure 4.

Illustration of how the mean-matched FF was computed. Data are from the MT-plaids dataset. $\boldsymbol{a}$. Spike rasters for the 46 trials (one per line) recorded from one MT neuron (127) for one stimulus condition (upwards-moving plaid). Shaded areas show 4 locations of the sliding window, which moved in $10 \mathrm{~ms}$ increments. For each window location, the spike count was computed for each trial. The mean and variance (across trials) of that count then contributed one data-point to the subsequent analysis (next panel). $\boldsymbol{b}$. The FF was computed based on scatterplots of the spike-count variance versus mean. Each scatterplot corresponds 
to a window in $a$. Each point plots data for one neuron/condition (red indicates the neuron/ condition from $a$ ). The orange line has unity slope: the expected variance-to-mean relationship for Poisson spiking. Data above the orange line is consistent with the presence of underlying-rate variability. Grey dots show all data. Gray lines are regression fits to all data (constrained to pass through zero, weighted according to the estimated SE of each variance measurement). Gray distributions are of mean counts. These appear to have different areas because of the vertical log scale. Black points are those preserved by meanmatching (methods). Black distributions are thus identical within the bin resolution. Black lines are regression slopes for the mean-matched data. $c$. The FF versus time. Arrows indicate time-points from the panels above. The gray trace (with flanking SEs) plots the 'raw' FF: the slope of the grey lines from panel $b$. The black trace plots the 'mean-matched' FF: the slope of the black lines. 
a

$\left.\begin{array}{ll}\frac{\infty}{w} & 5 \\ \frac{d}{\overline{ }} & \\ \frac{\text { के }}{3} & 3\end{array}\right]$

Mean rate (non-resp conds.)

के 1.5

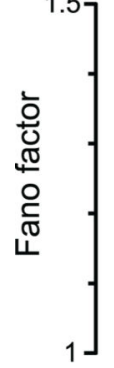
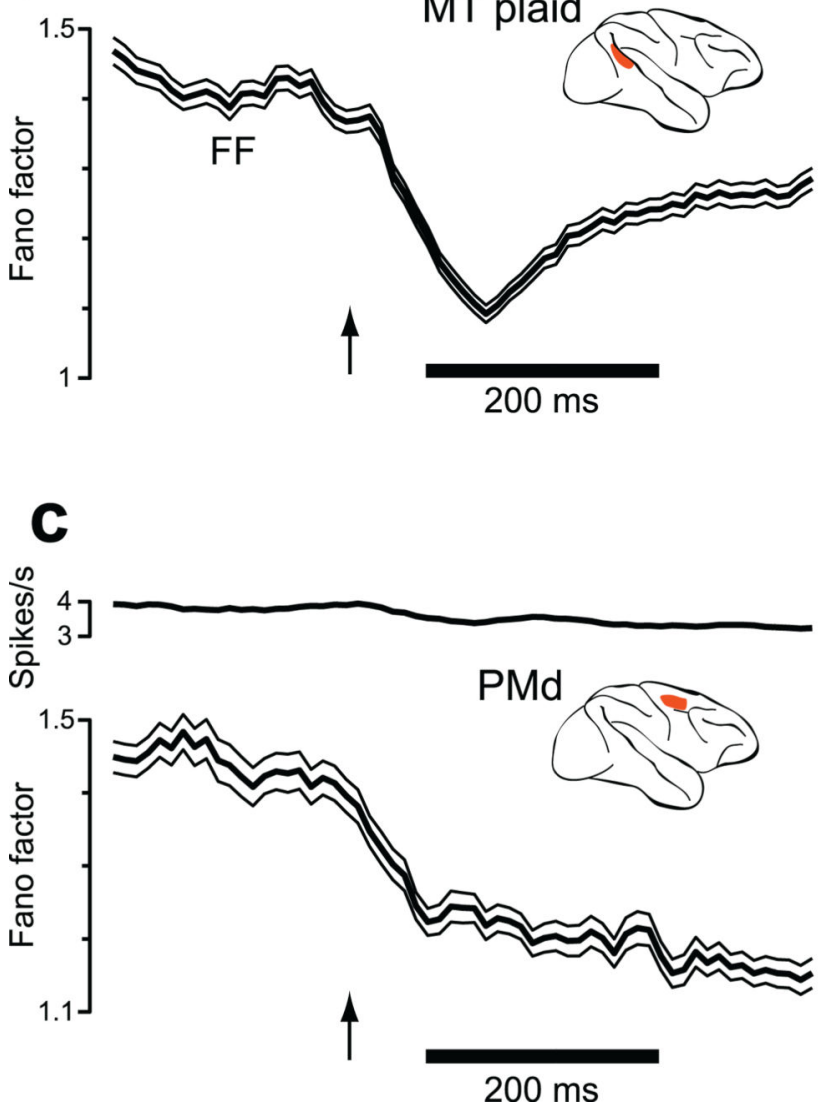

Figure 5. b
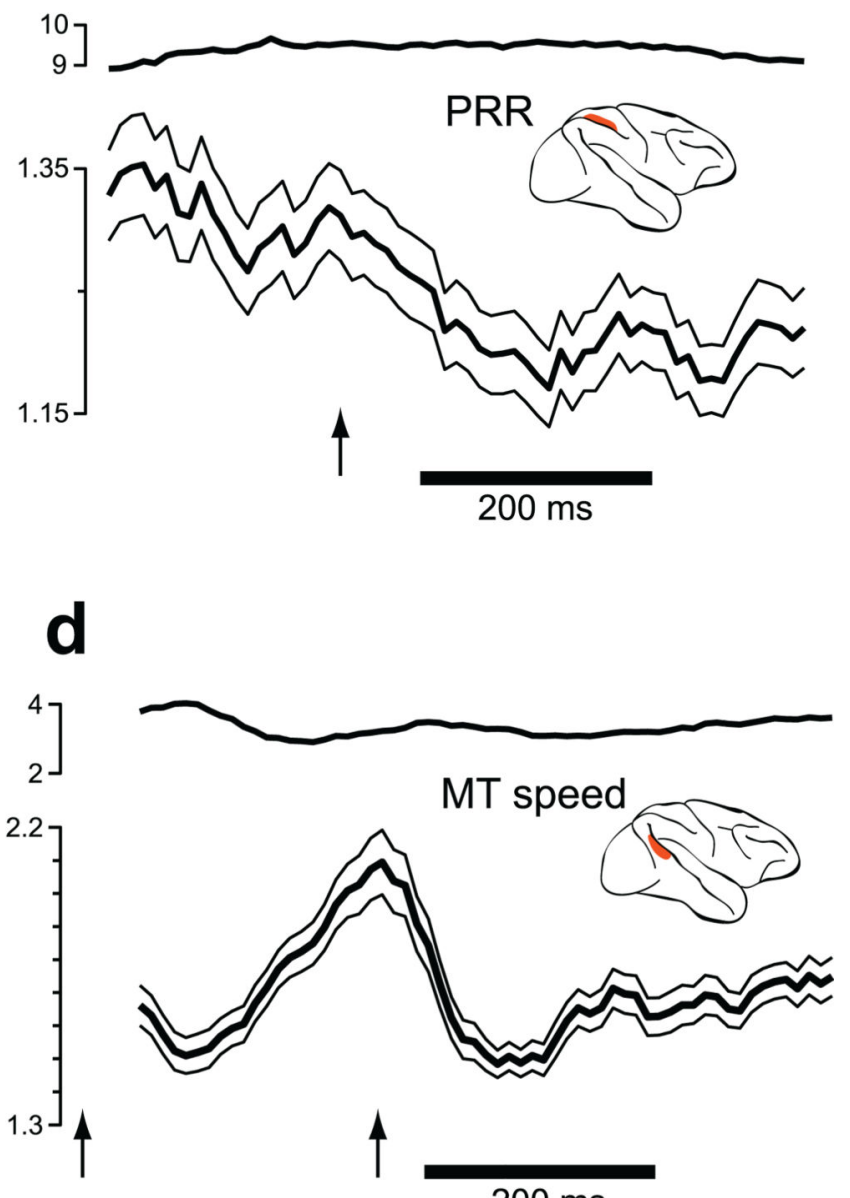

$200 \mathrm{~ms}$

Changes in the FF after restricting the analysis to neurons / conditions with little change in mean rate (e.g., non-preferred conditions). Of the original 'neuron-conditions' (the response of one neuron to one condition), this analysis preserved 28\% (MT, panel $a$ ), 49\% (PRR, panel $b$ ), 27\% (PMd, panel $c$ ), and 41\% (MT-speed, panel $d$ ). A $100 \mathrm{~ms}$ window (rather than $50 \mathrm{~ms}$ ) was employed to regain lost statistical power. The trace at the top of each panel shows the mean rate, averaged across all included neurons/conditions. The trace with flanking standard errors shows the FF, computed with no further mean matching. Arrows indicate stimulus onset. For the MT-speed dataset (panel d) the stimulus appeared at the very start of the record (first arrow) and began moving $256 \mathrm{~ms}$ later (second arrow). 



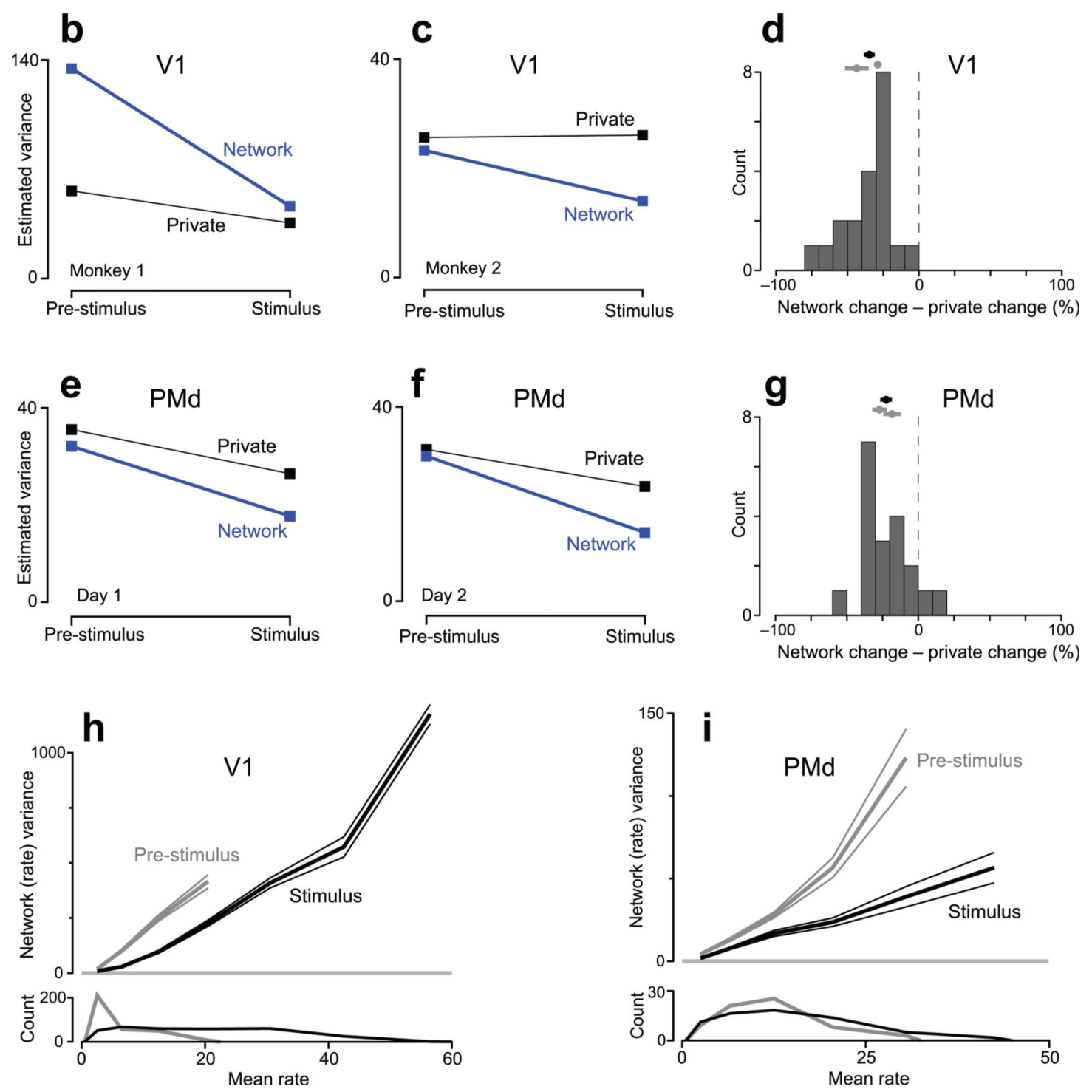

Figure 6.

Application of FA to data from V1 and PMd. See methods and Supplementary Figure 6 for a description of datasets. a. FA was applied to covariance matrices (\#neurons $\times$ \#neurons) of spike counts, taken in an analysis window that either ended at stimulus onset (pre-stimulus) or began just after stimulus onset (stimulus). The measured covariance matrix was approximated as the sum of a 'network' covariance matrix and a diagonal matrix of private noise. To produce the plots in $b-g$, network variances were averaged across the subset of neurons/conditions ( $48 \%$ and $30 \%$ for $\mathrm{V} 1,74 \%$ and $79 \%$ for PMd) whose distribution of 
mean rates was matched before and after stimulus onset (similar to Fig. 4). b. Estimated variances for one V1 dataset. Network variability declined more than private variability in both absolute $\left(\mathrm{p}<10^{-7}\right)$ and relative (percent of initial value, $\mathrm{p}<10^{-7}$ ) terms (paired t-tests across conditions). $\boldsymbol{c}$. similar plot for a V1 dataset from a second monkey: $\mathrm{p}<0.002$ (absolute) and $\mathrm{p}<0.002$ (relative). $\boldsymbol{d}$. Summary comparison for V1. Changes in variability (stimulus minus pre-stimulus) were expressed in percentage terms. Data to the left of zero indicate that network variability underwent the larger decline. The distribution includes all conditions and both datasets. The mean and standard error is given by the black symbol at top ( $\mathrm{p}<10^{-7}$ compared to zero, paired t-test). Gray symbols give individual means for each dataset. $\boldsymbol{e}$. Same as $b, c$ but for one PMd dataset (G20040123). Network variability declined more in absolute $(\mathrm{p}<0.005)$ and relative $(\mathrm{p}<0.001)$ terms. $f$. Similar plot for a second PMd dataset (G20040122): $\mathrm{p}<0.05$ (absolute) and $\mathrm{p}<0.02$ (relative). $g$. Summary comparison for PMd (distribution mean $\left.<0 ; \mathrm{p}<10^{-4}\right)$. $\boldsymbol{h}$. Relationship between mean firing rate and networklevel (shared firing-rate) variance. Data (same dataset as panel $b$ ) were binned by mean rate, and the average network variance $( \pm \mathrm{SE})$ was computed for each bin. This was done both pre-stimulus (gray) and after stimulus onset (black). The average was taken across neurons and conditions (each datum being averaged was, for one condition, one element of the blue diagonal in panel $a$ ). Distributions of mean rates are shown at bottom. The analysis in $b$ was based on the overlapping (mean-matched) portion of these distributions. $\boldsymbol{i}$. Similar plot for PMd (same dataset as panel $e$ ). 

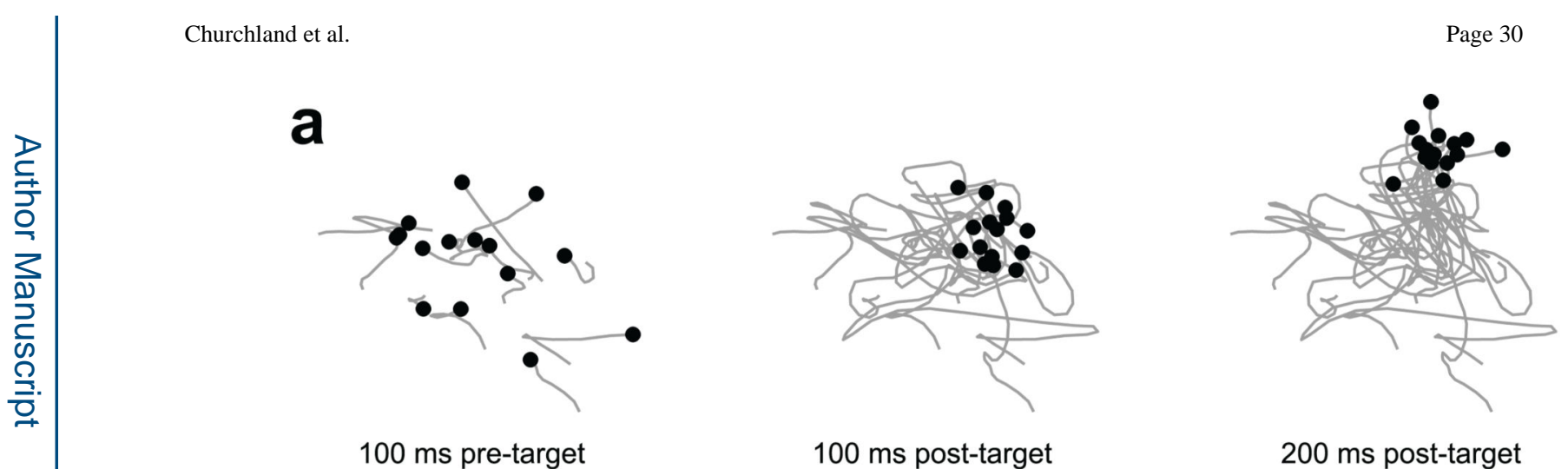

$100 \mathrm{~ms}$ pre-target

100 ms post-target

200 ms post-target

b

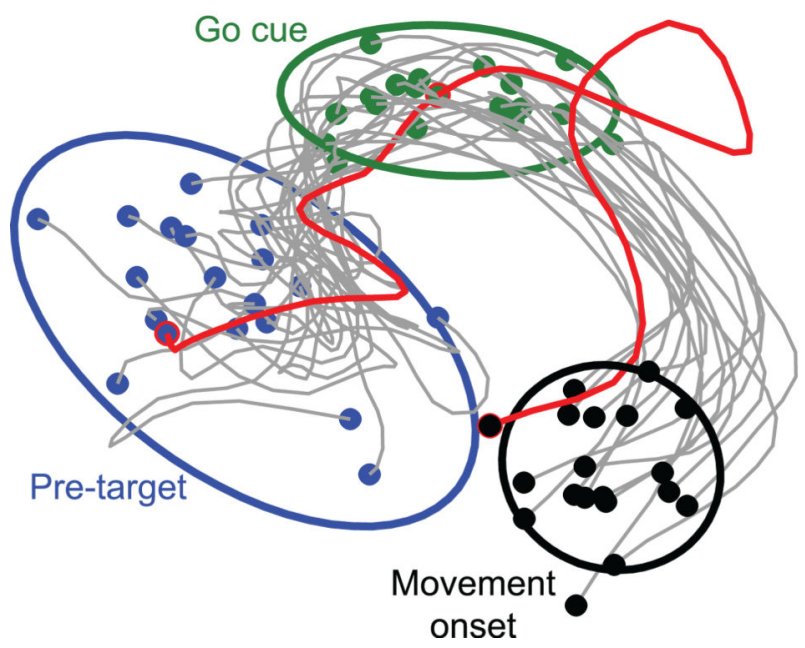

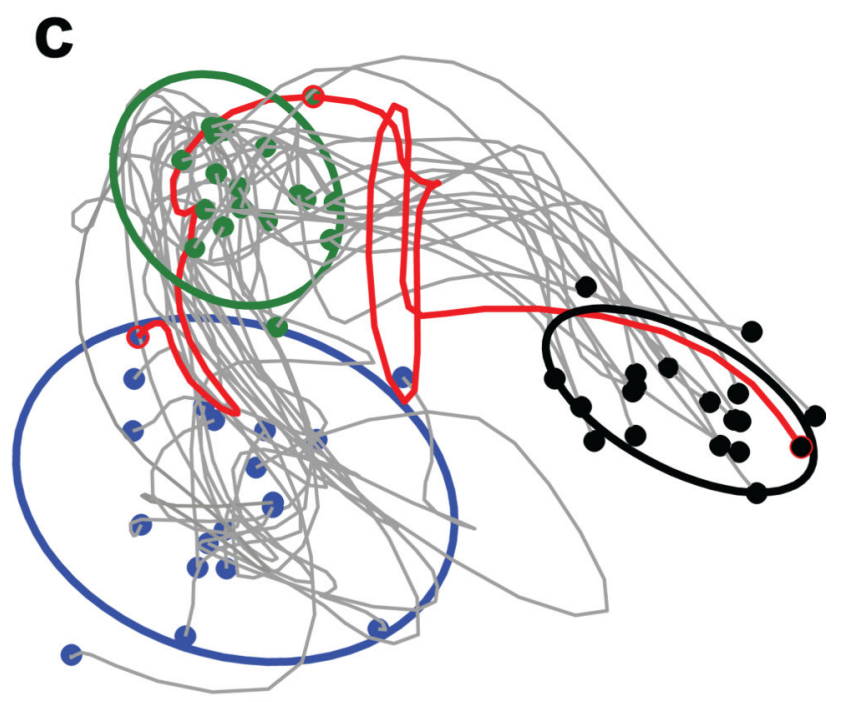

Figure 7.

Individual-trial neural trajectories, computed using GPFA. $\boldsymbol{a}$. Projections of PMd activity into a 2-dimensional state space. Each black point represents the location of neural activity on one trial. Gray traces show trajectories from $200 \mathrm{~ms}$ before target onset until the indicated time. The stimulus was a reach target $\left(135^{\circ}, 60 \mathrm{~mm}\right.$ distant), with no reach allowed until a subsequent go cue (see $b, c)$. Fifteen (of 47) randomly-selected trials are shown. Same dataset as in Figure 6e. $\boldsymbol{b}$. Trajectories are plotted until movement onset. Blue dots indicate $100 \mathrm{~ms}$ before stimulus (reach target) onset. No reach was allowed until after the go cue (green dots), 400-900 ms later. Activity between the blue and green dots thus relates to movement planning. Movement onset (black dots) was $\sim 300 \mathrm{~ms}$ after the go cue. For display, eighteen randomly-selected trials are plotted, plus one hand-selected trial (red, trialID211). Covariance ellipses were computed across all 47 trials. This is a 2-dimensional projection of a 10 dimensional latent space. In the full space, the black ellipse is far from the edge of the blue ellipse. This projection was chosen to accurately preserve the relative sizes (on a per dimension basis) of the true 10-dimensional volumes of the ellipsoids. G20040123 dataset. $\boldsymbol{c}$. Same presentation and target location as in $b$, but for data from another day's dataset (G20040122, red trial: trialID793). 


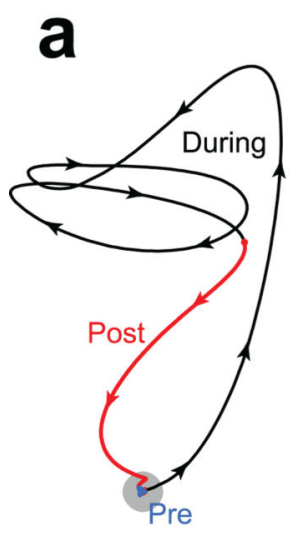

Mean

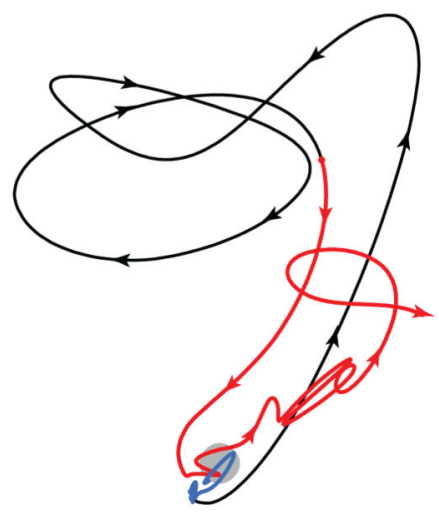

Trial 11

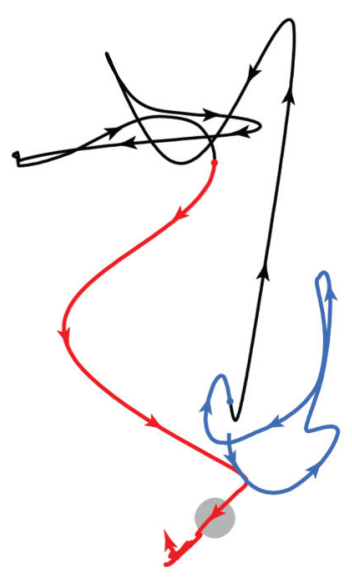

Trial 38

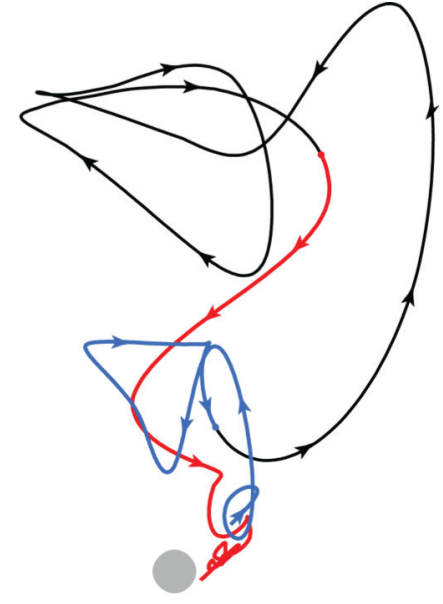

Trial 216


Figure 8.

Projections of V1 activity into a 2-dimensional space. Blue, black and red traces show activity before, during and after stimulus presentation (a drifting $45^{\circ}$ grating). Same dataset as Figure 6c. $a$. The mean trajectory and three trials picked by hand. The grey spot shows the average location of pre-stimulus activity. In a few cases (e.g., upper left portion of the rightmost panel), traces were moved very slightly apart to make it clear that they traveled in parallel rather than crossed. $\boldsymbol{b}$. Trajectories after data were shuffled to remove correlated variability. 25 randomly-selected trials are plotted (lighter traces) along with the mean (saturated traces). $c$. Same as in $b$, but for the original unshuffled data. 